\title{
Ultrafast Switching in Avalanche-Driven Ferroelectrics by Supersonic Kink Movements
}

\author{
Ekhard K. H. Salje,* Xiaofei Wang, Xiangdong Ding, and James F. Scott
}

Devices operating at $\mathrm{GHz}$ frequencies can be based on ferroelectric kinkdomains moving at supersonic speed. The kinks are located inside ferroelastic twin boundaries and are extremely mobile. Computer simulation shows that strong forcing generates velocities well above the speed of sound. Kinks are accelerated from $\boldsymbol{v}=\mathbf{0}$ continuously with Döring masses in the order of skyrmion masses under constant strain rates. Moving kinks emit phonons at all velocities, and the emission cones coincide with the Mach cones at supersonic speed. Kinks form avalanches with the emission of secondary kinks via a mother-daughter nucleation mechanism and may be observable in acoustic emission experiments. Supersonic kinks define a new type of material; while mobile domains are the key for ferroelastic and ferroelectric device applications at low frequencies, it is expected that fast kink movements replace such domain movements for materials applications at high frequencies.

approach that of transverse or longitudinal sound waves. This applies even for magnetic materials where coupling between magnetism and strain is usually weak. The Ramesh group observed fast switching of ferroelectric walls. ${ }^{[2]}$ Unfortunately, the limited time resolution of their experiment (switching time $220 \mathrm{ps}$ ) did not allow the exact determination of the characteristic time scale of the wall switching, but it can be estimated to be slightly below the speed of transverse sound waves. Coupled ultrafast polarization dynamics in ferroelectric nanolayers were reported by Korff Schmising et al. ${ }^{[3]}$ Jiang et al. ${ }^{[4,5]}$ demonstrated subpicosecond domain switching in $\mathrm{Pb}\left(\mathrm{Zr}_{0.35} \mathrm{Ti}_{0.65}\right) \mathrm{O}_{3}$. Their description may well relate to kink move-

\section{Introduction}

Ferroelastic switching is traditionally undertaken at time scales below the speed of sound, where pinning-depinning effects dominate the mobility of domain walls. Progress toward high frequency devices requires domain movements at supersonic speed. This is impossible for traditional ferroelectric switching that is based on the sideways movement of domain walls or, possibly, needle domains. We show in this paper that kinks inside domain walls can travel with supersonic speed under realistic external forcing. Such "walls inside walls" are hence a solution for the development of high-speed devices.

Coupling between the switchable parameter with elastic forces was already identified as the main obstacle to highspeed switching ${ }^{[1]}$ with high-energy losses when wall velocities

Prof. E. K. H. Salje, Dr. X. Wang, Prof. X. Ding

State Key Laboratory for Mechanical Behavior of Materials

Xi'an Jiaotong University

Xi'an 710049, China

E-mail: ekhard@esc.cam.ac.uk

Prof. E. K. H. Salje

Department of Earth Sciences

University of Cambridge

Cambridge CB2 3EQ, UK

Prof. J. F. Scott

School of Chemistry and School of Physics and Astronomy

St. Andrews University

St. Andrews KY169ST, UK

The ORCID identification number(s) for the author(s) of this article can be found under http://dx.doi.org/10.1002/adfm.201700367.

DOI: 10.1002/adfm.201700367 ments although the huge temperature range of these experiments may relate to classic domain movements, including the possibility of quantum critical behavior at sufficiently low temperatures. The high mobility of kink movement is geometrically akin to dislocation dynamics whereby dislocations show frequency dependent damping with limiting behavior of transonic and supersonic dislocations observed in computer experiments. ${ }^{[6-16]}$ Propagating shear cracks, ${ }^{[17-20]}$ mechanical twinning, ${ }^{, 21-23]}$ phase transition due to shock waves, ${ }^{[24]}$ dust particle motion in plasmas, ${ }^{[25]}$ ruptures, and earthquakes ${ }^{[26,27]}$ approach the speed of sound and in some cases may lead to supersonic wave propagation.

Kinks in ferroelastic twin walls can be continuously accelerated from a static position to supersonic speed in contrast to dislocations investigated in ref. [6]. Kinks are not necessarily ferroelectric but are often carrying secondary properties ${ }^{[28]} \mathrm{such}$ as ferroelectricity, ${ }^{[29]}$ polarity, ${ }^{[30-32]}$ (super-)conductivity, ${ }^{[3,34]}$ and magnetism. ${ }^{[35]}$ Complex wall structures can also form glass phases ${ }^{[36,37]}$ with ferroelectric properties. Kinks are singularities inside domain walls and we argue that such "walls in walls" are the key elements in the development of ferroic or superconducting (Josephson devices in ferroelastic superconductors) devices that operate on a picosecond time scale. Previous simulations of walls in ferroelastics showed already acoustic emission ${ }^{[38-41]}$ and speeds close to the sound barrier. A direct connection between traveling kinks and electric currents ${ }^{[42,43]}$ and dipolar switching ${ }^{[4-48]}$ was anticipated so that fast kink movements will typically induce equivalent polar front propagation. We show in this paper for the first time that supersonic movements in ferroic materials are related to such travelling kinks rather than any other of the complex movements of twin boundaries. 


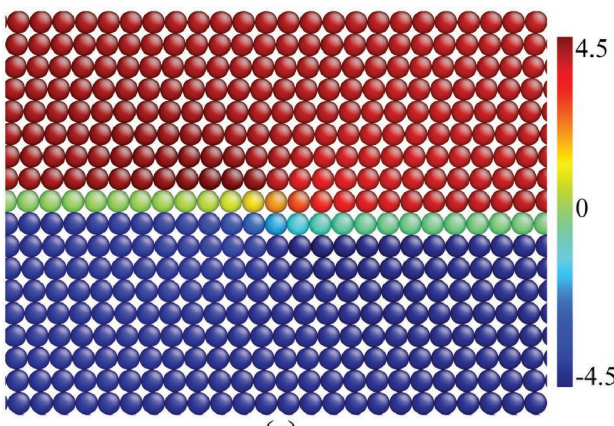

(a)

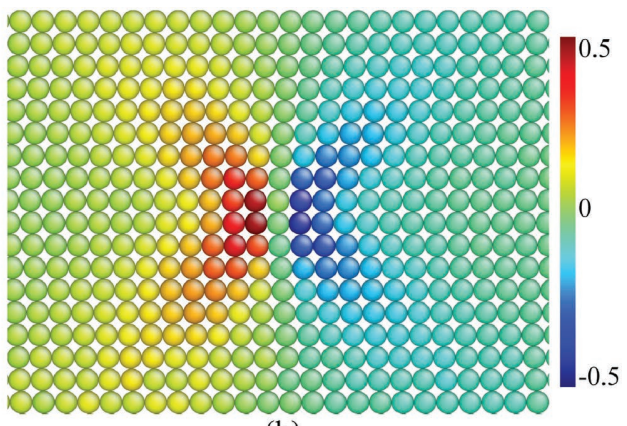

(b)

Figure 1. A static kink shown by a) the vertical shear angle and b) the horizontal shear angle. The strain field in (b) is similar to those of shear dislocations.

\section{Results and Discussion}

\subsection{Moving Kink inside a Ferroelastic Wall}

Figure 1 shows a static kink (or latch in 3D) as the fundamental element in our approach. This kink moves under shear stress. The direction of travel is determined by the energy gain by increasing the energetically favorable domain and reducing the unfavorable domain.

The overall location of the twin wall does not change; only the kink inside the twin wall moves until it hits the sample surface. The self-energy of a moving wall diverges in Landau-Ginzburg theory as $E \sim\left(1-v^{2} / c^{2}\right)^{-0.5}$, where $c$ is the relevant sound velocity and $v$ is the wall velocity. ${ }^{[47]}$ This divergence stems from the $1 \mathrm{D}$ character of the wall movement where the propagation direction coincides with the strain gradient as discussed in detail in ref. [47]. Kinks do not suffer from this singularity because the propagation and the strain gradient are rotated by $45^{\circ}$ with respect to each other (Figure $1 \mathrm{~b}$ ) so that any analytical description is intrinsically $2 \mathrm{D}$ avoiding the mass renormalization. Nevertheless, moving kinks also dissipate energy and this dissipation is the "stumbling block" for high-speed applications. Kinks within the most commonly discussed $\Phi 4$ model (the self energy is a 4th order polynomial) contain "wobbles" as internal degrees of freedom. ${ }^{[48]}$ Certain speeds in excess of the sound barrier are theoretically stable in $\Phi 4$ and generate emanating elastic waves during the propagation of the kink. ${ }^{[48-52]}$ Our molecular dynamics (MD) simulations of "realistic" ferroelastic kinks show that the situation is different: moving kinks emit secondary elastic waves for all velocities, in the supersonic scenario the emitted waves form Mach cones.

Ballistic movement of domain walls are characterized by the Döring mass, ${ }^{[53,54]}$ which measures the increase of the wall energy under acceleration. ${ }^{[55]}$ Positive and negative Döring masses are linked to the stability of driven magnetic domain walls. ${ }^{[56]}$ The kink or ledge becomes a localized region in 2D, topologically equivalent to a dot, which moves under the effect of an applied external strain (Figure 2).

We calculated the Döring mass at a strain rate of $10^{7} \mathrm{~s}^{-1}$ in which the change of velocity can reach to a steady state within our simulation time. As shown in Figure 2, the acceleration in the steady state is $9.3 \times 10^{9} \mathrm{~km} \mathrm{~s}^{-2}$ and the energy loss is $0.04992 \mathrm{eV}$. The kink moves over a distance of $379.3 \AA$. The kinetic mass (Döring mass) of the kink is hence $2.26 \times 10^{-26} \mathrm{~kg}$ or $13.64 \mathrm{amu}$. Our atomic mass is $10 \mathrm{amu}$ so that the Döring mass of the kink is slightly larger than that of one atom. The equivalent mass density of the ledge is expected to be in the order of $2 \times 10^{-26} \mathrm{~N} \mathrm{~kg} \mathrm{~m}^{-1}$, where $N$ is the number of crystallographic repetition lengths inside the ledge.

We compare this mass with magnetic domain walls and skyrmions and find that the mass of kinks are slightly less than skyrmions and magnetic walls (Figure 2). A typical magnetic mass density is $M \approx 10^{-9} \mathrm{~kg} \mathrm{~m}^{-2},{ }^{[55]}$ so that a patch of some $30 \times 30 \AA^{2}$ would have a similar mass of our kink. Inertia effects are usually neglected in metallic ferromagnets, as they are much smaller than eddy current dissipation. ${ }^{[57]}$ Zapperi et al. ${ }^{[58]}$ found a large negative mass $-7 \times 10^{-5} \mathrm{~kg} \mathrm{~m}^{-2}$. A skyrmion mass has been estimated with a lower limit of $8 \times 10^{-22} \mathrm{~kg},{ }^{[59]}$ which is much larger than domain wall masses as measured by Bedau et al. ${ }^{[60]}$ Their characteristic domain wall masses were $5.6 \times 10^{-25} \mathrm{~kg}$ for the transverse and $6.2 \times 10^{-24} \mathrm{~kg}$ for the longitudinal walls, and $6.3 \times 10^{-25} \mathrm{~kg}$ for the free and pinned vortex. Martinez and Jalil ${ }^{[61]}$ estimated a light Döring mass of a skyrmion as $\approx 10^{-25} \mathrm{~kg}$, which is slightly heavier than our Döring mass per unit cell in the kink.

The ballistic character of the kink movement is exemplified in kink movement when the increase of the external driving

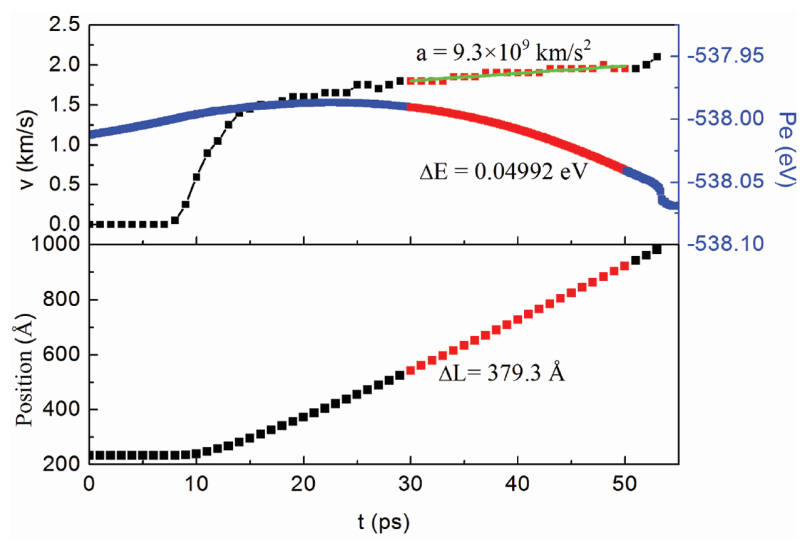

Figure 2. Accelerated movement of kinks at a strain rate of $10^{7} \mathrm{~s}^{-1}$. The kink is static for $7 \mathrm{ps}$ and then accelerated to a speed of near $1.5 \mathrm{~km} \mathrm{~s}^{-1}$. After 20 ps the kink shows a uniform acceleration of $9.3 \times 10^{9} \mathrm{~km} \mathrm{~s}^{-2}$. 
force is switched off (Figure 3). First, the kink is accelerated to velocities of $2.5,3.5$, and $6.3 \mathrm{~km} \mathrm{~s}^{-1}$, respectively, when the external strain is kept constant. In the subsonic regime $\left(<v_{\mathrm{T}[110]}, 4.38 \mathrm{~km} \mathrm{~s}^{-1}\right)$, the kink approximately continues the

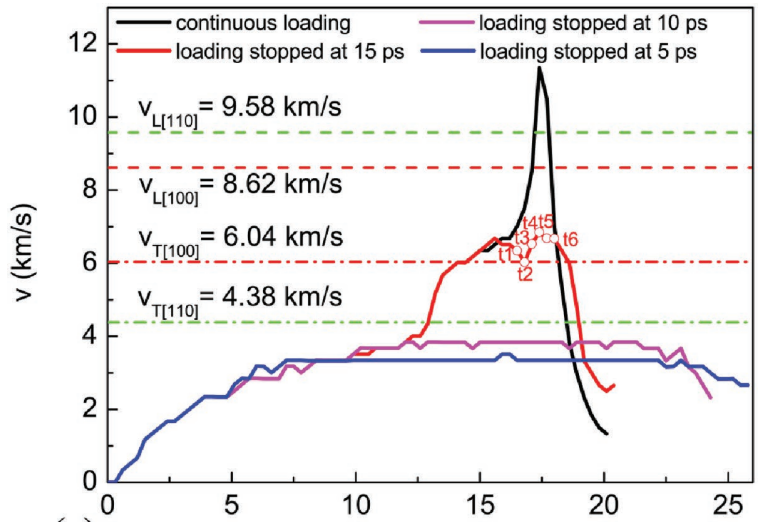

(a)

$t$ (ps)
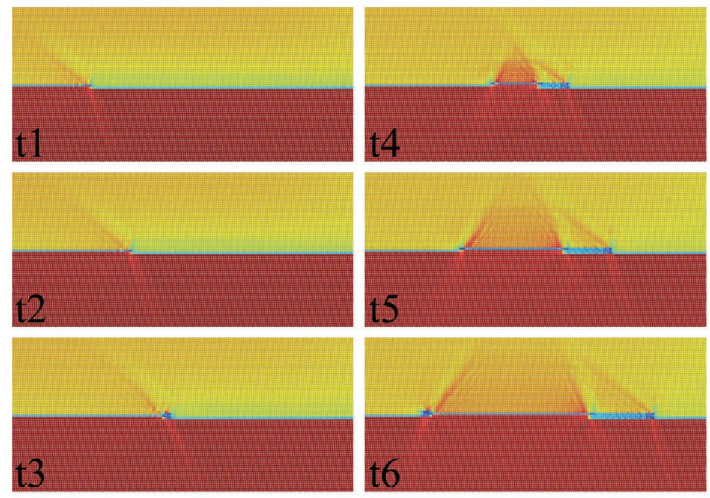

(b)

$-4.93$
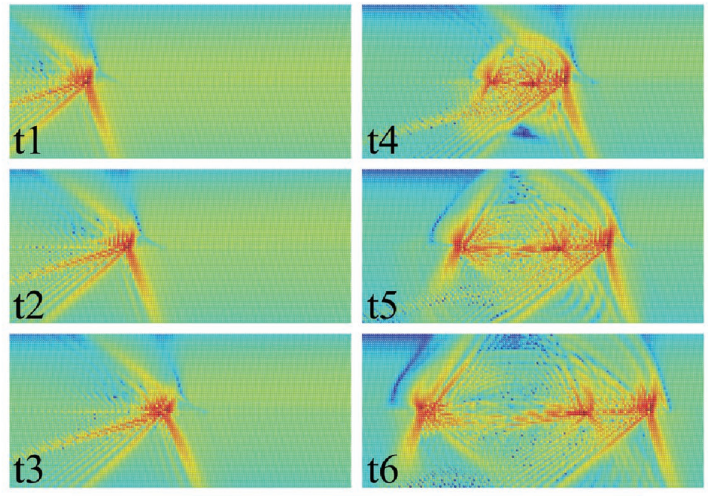

(c) -9.51

$-2.44$

Figure 3. Ballistic movement and the mother-daughter mechanism for kinks. a) Evolution of kink velocities with time. The kink is accelerated to velocities of $2.5,3.5$, and $6.3 \mathrm{~km} \mathrm{~s}^{-1}$, when loading is stopped at 5,10 , and $15 \mathrm{ps}$, respectively; in contrast, the evolution of kink velocity under continuous loading is shown in black line. b,c) mother-daughter mechanism for kinks with atoms colored by $\left|\theta_{\mathrm{v}}\right|-4.0+\theta_{\mathrm{h}}$ and $\log _{10}$ (kinetic energy), respectively. The mother kink travels with supersonic speed to the right. At $\mathrm{t} 3$ two daughter kinks nucleate and travel subsonically in opposite directions (t4-t6). The mother kink shows a supersonic cone (see movie in Supporting Information). movement at constant speed showing its ballistic characteristics (with weak dissipation, as shown in blue and pink line in Figure 3a). At speeds higher than the transverse sound velocity we find that the kink shows first some further acceleration and then a decrease of the speed (red line in Figure 3a). This decrease is related to the nucleation of pairs of secondary kinks, which travel in opposite directions in the adjacent layer above the pre-existing twin boundary. This mother-daughter mechanism $^{[17]}$ relates to moving kinks, which generate subsonic kinks whereby these kinks arrive as pairs (two daughters from each mother) and travel in opposite directions without changing the momentum of the mother kink.

The speed of the traveling kink under a strain rate of $10^{9} \mathrm{~s}^{-1}$ is shown in Figure 4. The kink is first accelerated from a static position to a speed of near $4 \mathrm{~km} \mathrm{~s}^{-1}$ near the sound velocity $v_{\mathrm{T}[110]}=4.38 \mathrm{~km} \mathrm{~s}^{-1}$. While progressing some 200 atomic positions on this plateau, the kink reaches the transverse sound velocity at $v_{\mathrm{T}[100]}=6.04 \mathrm{~km} \mathrm{~s}^{-1}$ and shows a second plateau at this speed. Further acceleration leads to a maximum velocity (11.35 $\mathrm{km} \mathrm{s}^{-1}$ ) before the end of the sample is reached which is greater than the longitudinal sound speed in [100] $\left(v_{\mathrm{L}[100]}=8.62 \mathrm{~km} \mathrm{~s}^{-1}\right)$ and [110] direction $\left(v_{\mathrm{L}[110]}=9.58 \mathrm{~km} \mathrm{~s}^{-1}\right)$. No plateau was found at the crossing of $v_{\mathrm{L}[100]}$ or $v_{\mathrm{L}[110]}$ (Figure 4). The kink profiles change during the movement. Phonons are emitted from the moving kink at all finite velocities while the static kink only induces a strain field similar to that of an edge dislocation. ${ }^{[62,63]}$ Strain related to the emitted sound at subsonic speeds is the lowest energy excitation of a transverse sound wave along [110], showing a backward cone with an opening angle of $45^{\circ}$. This signal remains strong for all subsonic speeds. Additional waves are seen when the speed exceeds transverse sound velocity $\left(v_{\mathrm{T}[110]}\right.$ and $\left.v_{\mathrm{T}[100]}\right)$ where the cone angle $\alpha$ approaches $31^{\circ}$ (t4, Figure 5) at a slightly higher velocity of $8.51 \mathrm{~km} \mathrm{~s}^{-1}$. In the Mach construction, we find $\sin \alpha=c / v$, where $v$ is the speed of the propagating kink

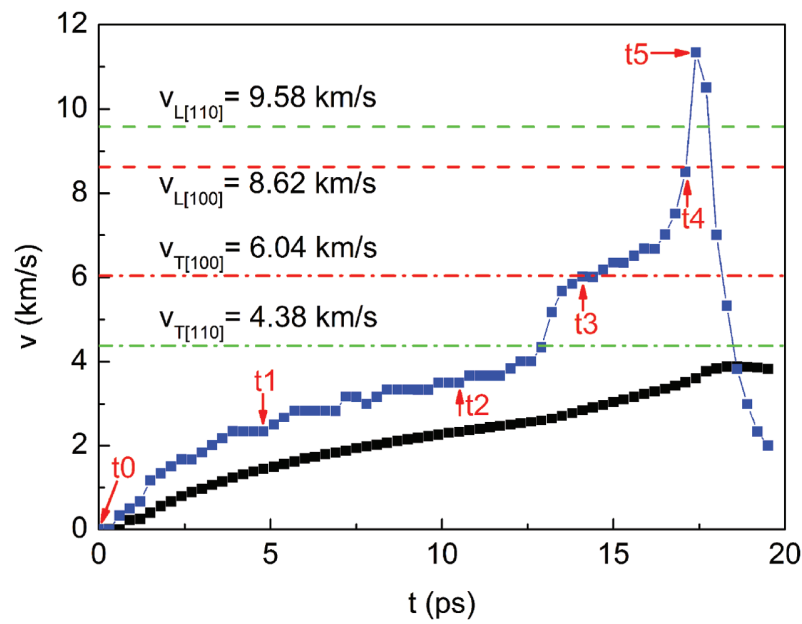

Figure 4. Velocity evolution of the kink at strain rate of $10^{9} \mathrm{~s}^{-1}$. The black curve gives the speed $x / t$, the blue curve is the local speed $\mathrm{d} x / \mathrm{d} t$, where $x$ is the traveled position and $t$ is the time. The kink is accelerated from a static position (t0) to a maximum local velocity of $11.35 \mathrm{~km} \mathrm{~s}^{-1}(\mathrm{t} 5)$ before it hits the surface. Note that supersonic speeds do not require high-speed initial conditions, as is the case for dislocations. ${ }^{[6]}$ The various sound velocities are shown by horizontal lines. 

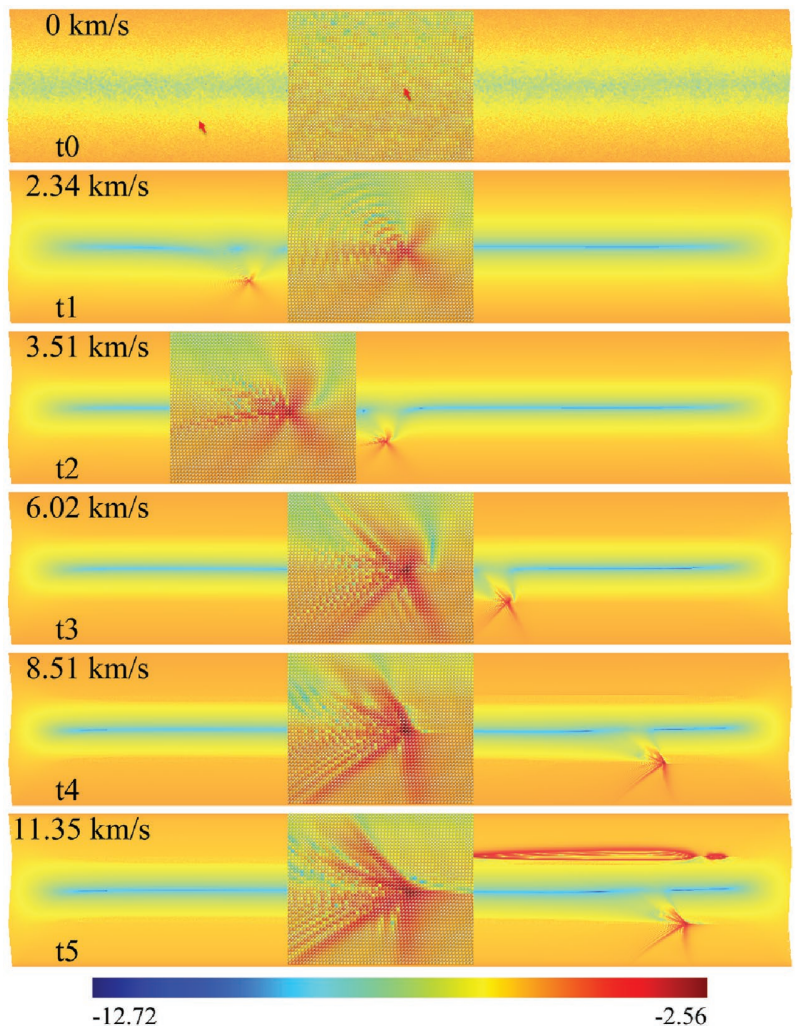

Figure 5. Phonon cones for various speeds colored by $\log _{10}$ (kinetic energy). Starting from static (t0), the kink is accelerated to subsonic $(\mathrm{t} 1-\mathrm{t} 2)$, transonic ( $\mathrm{t} 3-\mathrm{t} 4)$, and supersonic ( $\mathrm{t} 5$ ) regimes, the inserted figures at $\mathrm{t} 1-\mathrm{t} 5$ show the corresponding wave emissions at different speeds. The red arrow in the top panel indicates the position of the static kink.

and $c$ is the wave velocity. For a kink speed of $8.51 \mathrm{~km} \mathrm{~s}^{-1}$ we find $c=4.38 \mathrm{~km} \mathrm{~s}^{-1}$, which is exactly the transverse sound speed in the [110] direction. Simultaneously, the halo of the $v_{\mathrm{L}[100]}$ velocity $\left(8.62 \mathrm{~km} \mathrm{~s}^{-1}\right)$ is clearly visible.

\subsection{Experimental Evidence for High-Speed Ferroelectric Switching}

Metaxas et al. speculated in 2007 that three distinct regimes of domain wall motion are generally predicted: creep, depinning, and hydrodynamic flow. ${ }^{[64]}$ However, they emphasized that the flow regime had not been experimentally accessible because it requires such large nonlinear stresses that samples might not physically survive. Such a hydrodynamic flow regime is, however, well studied in ferroelectric smectic liquid crystals and involves several instabilities related to folding, ${ }^{[65]}$ including the Parodi instability and the Bobylev-Pikin instability. A general review of such nonequilibrium patterning has been given by Cross and Hohenberg. ${ }^{[66]}$ Size reduction was stimulated similar to the simulations described in the section above, for the special case of ferroelectric nanodomains nestled inside slightly larger ferroelastic domains were investigated. ${ }^{67,68]}$ These systems exhibit wrinkling and folding, chevron structures at angles different from $90^{\circ}$, and nonexponential relaxation motions. ${ }^{[68]}$ The fact that they exist in super-domain configurations arises from the fact that the ferroelectric domains can organize into blocks such that average polarization $\langle P\rangle=0$, but these blocks have a nonzero net strain $\langle S\rangle$, which can also be made to average zero by clustering blocks into a superdomain. Note that the ferroelectric blocks are always inside the ferroelastic blocks and never vice versa; this is because the polarization coherence length is screened but strain is unscreened. Our results show that ferroelectric kinks in ferroelastic walls fulfill the same conditions but also reduce the dimensionality of the problem. In this paper, we showed that a similar distinction has to be made between lateral twin wall movements, which cannot be supersonic in classic theory, and movements of kinks inside domain walls, which are supersonic under sufficient forcing.

Notable is the prediction that the folding instabilities occur with a dependence upon film thickness $d$ as $d^{4 / 9},{ }^{[69,70]}$ which is not yet confirmed experimentally but could be measured with wedge-shaped films. We also remind readers that domain walls have been driven to produce a nonlinear flow by applied voltages, ${ }^{[71]}$ but thus far only at low velocities. Some ferroelastic domains that appear to be the result of nonlinear viscous are thixotropic, like toothpaste (viscosity decreases with time at constant stress), or superplastic, or exhibit overshoot. A general review is given in ref. [72] and the specific application of Mittag-Leffler models of relaxation to dielectrics, with power laws, in ref. [73]. The fastest switching observed for ferroelectric domains is about $1-2 \mathrm{ps}^{[3-5]}$ and the lowest coercive voltages, about $250 \mathrm{mV}$, both across a $6 \mathrm{~nm}$ film. ${ }^{[74]}$ If we assume that a 2 ps time involves nucleation entirely at one electrode, then the wall velocity is $6 \mathrm{~nm} / 2 \mathrm{ps}=3 \mathrm{~km} \mathrm{~s}^{-1}$, which is very nearly the speed of sound. So the ultrasonic simulations are plausible, particularly as they are supported by the observation of acoustic phonon emission during switching. ${ }^{[75]}$ As with the original experiments of Demokritov et al., ${ }^{[7,77]}$ it would be highly desirable to measure the emission angle of these acoustic phonons in Figure 5; as with bow waves or Cerenkov radiation, this angle would give a precise value of supersonic wall velocity, as has been shown in our simulations. Although we cannot yet measure directly this ultrafast switching, Gruverman et al. have shown how to do so with a progressive series of single-frame piezoresponse force microscope measurements that are reassembled like a slow-motion video. ${ }^{[78]}$ Such data should be available in the near future. In addition, we have strong indirect evidence for the requisitive high-strain situation in the form of domain wall period-doubling. Period doubling of ferroelastic domain widths is predicted at high stress. ${ }^{[79]}$ This has been observed in the time domain in unrelated work on illuminated microcrystals in fluids by one of us, with a sharp threshold at modest continuous wave laser power. ${ }^{[0]}$ In additions, high-speed kink movements were claimed from the experimental observation of high frequency resonances in resonant ultrasonic spectroscopy. ${ }^{[81-83]}$

\section{Conclusion}

In summary, we have shown that high-speed switching becomes possible if the moving domain boundary is not the ferroelectric wall but a kink inside a ferroelastic wall. This opens the way to the use of unforeseen materials for ferroelectric device applications. Prototypic materials are $\mathrm{CaTiO}_{3}$, 
which has the desired ferroelectric component of its ferroelastic walls ${ }^{[30]}$ or $\mathrm{LiNbO}_{3}$, which contains large number of kinks inside ferroelectric walls. ${ }^{[42]}$ Another example is $\mathrm{SrTiO}_{3}$, which contains many ferroelastic walls and, expectedly, kinks, which may carry polarity. ${ }^{[45]}$ Another example is $\mathrm{LaAlO}_{3}$, where polarity was found in tweed structures ${ }^{[43]}$ where moving kinks may be possible. We also connect our research with simulated ferroelectric switching in bulk ${ }^{[84]}$ and switching in thin films. ${ }^{[85]}$ Preliminary experiments exist in the literature, showing rapid domain flow at high stress levels.

\section{Experimental Section}

Our computer simulations were based on the standard ferroelastic model; the potential energy $U(r)$ was composed of three interactions, the first-nearest atomic interactions $U_{\mathrm{NN}}=2(r-1)^{2}(0.8 \leq r \leq 1.2)$, the second-nearest atomic interactions $U_{\mathrm{NNN}}=-(r-\sqrt{2})^{2}+200(r-\sqrt{2})^{4}$ $(1.207 \leq r \leq 1.621)$, and the third-nearest interactions $U_{\text {NNNN }}=$ $-0.1(r-2)^{4}(1.8 \leq r \leq 2.2)$, where $r$ is atomic distance vector. The shear angle was the order parameter in this model. The equilibrium unit cell was a parallelogram with a shear angle of $4^{\circ}$. The equilibrium lattice constant $a=1 \AA$ and atomic mass $M=10$ amu were set. Extensions of this generic model with one atom per unit cell were discussed in refs. [31] and [32]. There it was shown that the relatively high moduli for one atom models decreased for more complex structures to values typical for perovskite structures. Free boundary conditions were adopted and twinned sandwich structure containing two pre-existing horizontal twin boundaries as the initial configuration. The size of our 2D simulation was $1000 a \times 202 a$. The ratio of the height of the switchable intermediate layer to the total sample was fixed to be 0.5 . The system was first relaxed with a conjugate gradient refinement procedure at the beginning of the simulations. Then MD was performed to anneal the configuration at a given temperature for $3 \times 10^{6}$ time steps. No microstructures developed, except for some surface relaxations, during this procedure. Finally, at the top and bottom several layers of atoms were fixed rigidly and then sheared with a constant shear strain rate in a canonical ensemble. All MD simulations were performed with the LAMMPS code and the Nose-Hoover thermostat was used to hold the sample temperature constant at $0.001 \quad K \cdot{ }^{[86-89]}$ The movement of the kinks was adiabatic and driven by a constant strain rate, in contrast to jin et al. ${ }^{[90]}$ where the strain reduced over time. The elastic moduli of the model were $c_{11}=1233.9 \mathrm{GPa}, c_{22}=1223.2 \mathrm{GPa}$, $c_{12}=598.6 \mathrm{GPa}$, and $c_{66}=606.3 \mathrm{GPa}$. The moduli corresponded to the pseudo cubic symmetry $\left(c_{11}=c_{22}, c_{12}=c_{66}\right)$ and the central forces of the model $\left(c_{12}=c_{11} / 2\right)$. The speed of sound in the [100] direction was $\left(c_{44} / \rho\right)^{1 / 2}=6.04 \mathrm{~km} \mathrm{~s}^{-1}$ for transverse waves and $\left(c_{11} / \rho\right)^{1 / 2}=8.62 \mathrm{~km} \mathrm{~s}^{-1}$ for longitudinal waves, where $\rho$ is the density. The secondary waves velocities in the [110] direction were $\left(\left(c_{11}+c_{12}+2 c_{44}\right) / 2 \rho\right)^{1 / 2}=9.58 \mathrm{~km} \mathrm{~s}^{-1}$ for longitudinal and $\left(\left(c_{11}-c_{12}\right) / 2 \rho\right)^{1 / 2}=4.38 \mathrm{~km} \mathrm{~s}^{-1}$ for transverse waves.

\section{Supporting Information}

Supporting Information is available from the Wiley Online Library or from the author.

\section{Acknowledgements}

The authors appreciate the support of the Natural Science Foundation of China (51320105014, 51621063, and 51231008) and 111 project (B06025). E.K.H.S. is also grateful to the Engineering and Physical
Sciences Research Council (EP/K009702/1) and the Leverhulme Foundation (RPG-2012-564) for support.

\section{Keywords}

acoustic emission, avalanche formation, cracking noise, ferroelectric switching, ferroelastic walls, kinks, supersonic wall movements

Received: January 20, 2017

Revised: February 19, 2017 Published online:

[1] S. O. Demokritov, A. I. Kirilyuk, N. M. Kreines, V. I. Kudinov, V. B. Smirnov, M. V. Chetkin, J. Magn. Magn. Mater. 1991, 102, 339.

[2] J. Li, B. Nagaraj, H. Liang, W. Cao, C. H. Lee, R. Ramesh, Appl. Phys. Lett. 2004, 84, 1174.

[3] C. V. Korff Schmising, M. Bargheer, M. Kiel, N. Zhavoronkov, M. Woerner, T. Elsaesser, I. Vrejoiu, D. Hesse, M. Alexe, Phys. Rev. Lett. 2007, 98, 257601.

[4] A. Jiang, H. Lee, C. Hwang, J. F. Scott, Adv. Funct. Mater. 2012, 22 192.

[5] A. Jiang, Z. Chen, W. Hui, D. Wu, J. F. Scott, Adv. Funct. Mater. 2012, 22, 2148.

[6] P. Gumbsch, H. Gao, Science 1999, 283, 965.

[7] P. Gumbsch, H. Gao, J. Comput.-Aided Mater. Des. 1999, 6, 137

[8] E. Bitzek, P. Gumbsch, Mater. Sci. Eng., A 2004, 11, 387.

[9] H. Koizumi, H. O. K. Kirchner, T. Suzuki, Phys. Rev. B 2002, 65, 214104.

[10] H. Koizumi, T. Suzuki, Mater. Sci. Eng., A 2005, 400-401, 76.

[11] D. L. Olmsted, L. G. Hector Jr., W. A. Curtin, R. J. Clifton, Modell. Simul. Mater. Sci. Eng. 2005, 13, 371.

[12] J. Marian, W. Cai, V. V. Bulatov, Nat. Mater. 2004, 3, 158.

[13] J. A. Y. Vandersall, B. D. Wirth, Philos. Mag. 2004, 84, 3755.

[14] S. Q. Shi, H. C. Huang, C. H. Woo, Comput. Mater. Sci. 2002, 23, 95.

[15] H. Gao, Y. Huang, P. Gumbsch, A. J. Rosakis, J. Mech. Phys. Solids 1999, 47, 1941.

[16] P. Rosakis, Phys. Rev. Lett. 2001, 86, 95.

[17] F. F. Abraham, H. J. Gao, Phys. Rev. Lett. 2000, 84, 3113.

[18] A. J. Rosakis, O. Samudrala, D. Coker, Science 1999, 284, 1337.

[19] K. Xia, A. J. Rosakis, H. Kanamori, Science 2004, 303, 1859.

[20] M. J. Buehler, F. F. Abraham, H. J. Gao, Nature 2003, 426, 141.

[21] P. Rosakis, H. Tsai, Int. J. Solids Struct. 1995, 32, 2711.

[22] J. Červ, M. Landa, A. Machova, Scr. Mater. 2000, 43, 423.

[23] J. Bosansky, T. Smida, Mater. Sci. Eng., A 2002, 323, 198.

[24] K. Kadau, T. C. Germann, P. S. Lomdahl, B. L. Holian, Science 2002, 296, 1681

[25] V. Nosenko, J. Goree, Z. W. Ma, D. H. E. Dubin, A. Piel, Phys. Rev. E 2003, 68, 056409

[26] A. Sagy, Z. Reches, J. Fineberg, Nature 2002, 418, 310.

[27] E. Olson, R. M. Allen, Nature 2005, 438, 212.

[28] E. K. H. Salje, ChemPhysChem 2010, 11, 940.

[29] E. K. H. Salje, S. Li, Z. Zhao, P. Gumbsch, X. Ding, Appl. Phys. Lett. 2015, 106, 212907

[30] S. V. Aert, S. Turner, R. Delville, D. Schryvers, G. V. Tendeloo, E. K. H. Salje, Adv. Mater. 2012, 24, 523.

[31] E. K. H. Salje, S. Li, M. Stengel, P. Gumbsch, X. Ding, Phys. Rev. B 2016, 94, 024114

[32] T. Zykova-Timan, E. K. H. Salje, Appl. Phys. Lett. 2014, 104, 082907.

[33] J. Seidel, P. Maksymovych, Y. Batra, A. Katan, S.-Y. Yang, Q. He, A. P. Baddorf, S. V. Kalinin, C.-H. Yang, J.-C. Yang, Y.-H. Chu, E. K. H. Salje, H. Wormeester, M. Salmeron, R. Ramesh, Phys. Rev. Lett. 2010, 105, 197603. 
[34] A. Aird, E. K. H. Salje, J. Phys.: Condens. Matter 1998, 10, L377.

[35] M. A. Carpenter, E. K. H. Salje, C. J. Howard, Phys. Rev. B 2012, 85, 224430.

[36] E. K. H. Salje, M. A. Carpenter, Phys. Status Solidi B 2015, 252, 2639.

[37] E. K. H. Salje, X. Ding, O. Aktas, Phys. Status Solidi B 2014, 251, 2061.

[38] E. K. H. Salje, X. Wang, X. Ding, J. Sun, Phys. Rev. B 2014, 90, 064103.

[39] L. Zhang, E. K. H. Salje, X. Ding, J. Sun, Appl. Phys. Lett. 2014, 104, 162906.

[40] E. K. H. Salje, Annu. Rev. Mater. Res. 2012, 42, 265.

[41] R. J. Harrison, E. K. H. Salje, Appl. Phys. Lett. 2010, 97, 021907.

[42] J. Gonnissen, D. Batuk, G. F. Nataf, L. Jones, A. M. Abakumov, S. V. Aert, D. Schryvers, E. K. H. Salje, Adv. Funct. Mater. 2016, 26, 7599.

[43] E. K. H. Salje, M. Alexe, S. Kustov, M. C. Weber, J. Schiemer, G. F. Nataf, J. Kreisel, Sci. Rep. 2016, 6, 27193.

[44] E. K. H. Salje, Z. Zhao, X. Ding, J. Sun, Am. Mineral. 2013, 98, 1449.

[45] E. K. H. Salje, J. F. Scott, Appl. Phys. Lett. 2014, 105, 252904.

[46] E. K. H. Salje, O. Aktas, M. A. Carpenter, V. V. Laguta, J. F. Scott, Phys. Rev. Lett. 2013, 111, 247603.

[47] E. K. H. Salje, Phase Transitions in Ferroelastic and Co-Elastic Crystals, Cambridge University Press, Cambridge, UK 1993.

[48] G. Kalbermann, J. Phys. A: Math. Gen. 2004, 37, 11603.

[49] I. V. Barashenkov, O. F. Oxtoby, Phys. Rev. E 2009, 80, 026608.

[50] O. F. Oxtoby, I. V. Barashenkov, Phys. Rev. E 2007, 76, 036603.

[51] S. V. Dmitriev, A. Khare, P. G. Kevrekidis, A. Saxena, L. Hadzievski, Phys. Rev. E 2008, 77, 056603.

[52] R. B. Tew, J. A. D. Wattis, J. Phys. A: Math. Gen. 2001, 34, 7163.

[53] W. Döring, Z. Naturforsch. A 1948, 3, 373.

[54] A. Hubert, R. Schäfer, Magnetic Domains, Springer, Berlin, Germany 1998

[55] G. T. Rado, R. W. Wright, W. H. Emerson, Phys. Rev. 1950, 80, 273.

[56] E. Magyari, H. Thomas, Z. Phys. B 1985, 59, 167

[57] G. Bertotti, Hysteresis in Magnetism, Academic Press, San Diego, CA 1998.

[58] S. Zapperi, C. Castellano, F. Colaiori, G. Durin, Nat. Phys. 2005, 1, 46.

[59] F. Büttner, C. Moutafis, M. Schneider, B. Krüger, C. M. Günther, J. Geilhufe, C. V. Korff Schmising, J. Mohanty, B. Pfau, S. Schaffert, A. Bisig, M. Foerster, T. Schulz, C. A. F. Vaz, J. H. Franken, H. J. M. Swagten, M. Kläui, S. Eisebitt, Nat. Phys. 2015, 11, 225.

[60] D. Bedau, M. Kläui, S. Krzyk, U. Rüdiger, G. Faini, L. Vila, Phys. Rev. Lett. 2007, 99, 146601

[61] J. C. Martinez, M. B. A. Jalil, IEEE Trans. Magn. 2015, 51, 1100204.
[62] J. P. Hirth, J. Lothe, Theory of Dislocations, Wiley, NewYork, NY 1982.

[63] D. C. Parfitt, C. L. Bishop, M. R. Wenman, R. W. Grimes, J. Phys.: Condens. Matter 2010, 22, 175004.

[64] P. J. Metaxas, J. P. Jamet, A. Mougin, M. Cormier, J. Ferre, V. Baltz, B. Rodmacq, B. Dieny, R. L. Stamps, Phys. Rev. Lett. 2008, 99, 2172083.

[65] L. M. Blinov, J. Phys. Colloq. 1979, 40, C3-247.

[66] M. C. Cross, P. C. Hohenberg, Rev. Mod. Phys. 1993, 65, 851.

[67] J. F. Scott, J. Phys.: Condens. Matter 2015, 27, 492001.

[68] J. F. Scott, D. M. Evans, J. M. Gregg, A. Gruverman, Appl. Phys. Lett. 2016, 109, 042901.

[69] R. Huang, J. Mech. Phys. Solids 2008, 56, 3315.

[70] L. Feigl, P. Yudin, I. Stolichnov, T. Sluka, K. Shapovalov, M. Mtebwa, C. S. Sandu, X.-K. Wei, A. K. Tagantsev, N. Setter, Nat. Commun. 2014, 5, 4677

[71] J. R. Whyte, R. G. P. McQuaid, P. Sharma, C. Canalias, J. F. Scott, A. Gruverman, J. M. Gregg, Adv. Mater. 2013, 25, 293.

[72] S. Brazovskii, T. Nattermann, Adv. Phys. 2004, 53, 177

[73] E. de Oliveira, F. Mainardi, J. Vaz, Eur. Phys. J.: Spec. Top. 2011, 193, 161.

[74] R. Ramesh, private communication, November 2016; Ferroics 2017, Williamsburg, VA, February 2017.

[75] E. Salje, E. Dul'kin, M. Roth. Appl. Phys. Lett. 2015, 106, 152903.

[76] S. O. Demokritov, A. I. Kirilyuk, N. M. Kreines, V. I. Kudinov, V. B. Smirnov, M. V. Chetkin, J. Magn. Magn. Mater. 1991, 102, 339.

[77] S. O. Demokritov, N. M. Kreines, V. I. Kudinov, JETP Lett. 1985, 41, 46.

[78] A. Gruverman, D. Wu, J. F. Scott, Phys. Rev. Lett. 2008, 100, 097601.

[79] I. Tsatskis, E. K. H. Salje, V. Heine, J. Phys.: Condens. Matter 1994, 6, 11027.

[80] K. W. McGregor, R. A. O'Sullivan, J. F. Scott, Physica A 1998, 254, 358.

[81] M. A. Carpenter, Z. Zhang, Geophys. J. Int. 2011, 186, 279

[82] M. A. Carpenter, A. Buckley, P. A. Taylor, T. W. Darling, J. Phys.: Condens. Matter 2010, 22, 035405.

[83] M. A. Carpenter, J. Phys.: Condens. Matter 2015, 27, 263201.

[84] S. Liu, I. Grinberg, A. M. Rappe, Nature 2016, 534, 360.

[85] J. Y. Jo, S. M. Yang, T. H. Kim, H. N. Lee, J.-G. Yoon, S. Park, Y. Jo, M. H. Jung, T. W. Noh, Phys. Rev. Lett. 2009, 102, 045701

[86] E. K. H. Salje, X. Ding, Z. Zhao, Phys. Rev. B 2011, 83, 104109.

[87] S. Plimpton, J. Comput. Phys. 1995, 117, 1.

[88] S. Nose, J. Chem. Phys. 1984, 81, 511.

[89] W. G. Hoover, Phys. Rev. A 1985, 31, 1695.

[90] Z. Jin, H. Gao, P. Gumbsch, Phys. Rev. B 2008, 77, 094303. 